\title{
CORRECTION
}

\section{Correction to: Epidemiology and patient predictors of infection and sepsis in the prehospital setting}

Daniel J. Lane ${ }^{1,2^{*}} \mathbb{D}$, Hannah Wunsch 1,3,4,5, Refik Saskin ${ }^{1}$, Sheldon Cheskes ${ }^{2,6,7}$, Steve Lin ${ }^{1,2,8}$, Laurie J. Morrison²,8, Christopher J. Oleynick ${ }^{9}$ and Damon C. Scales ${ }^{1,2,3,4}$

(C) 2020 Springer-Verlag GmbH Germany, part of Springer Nature

\section{Correction to: Intensive Care Med https://doi.} org/10.1007/s00134-020-06093-4

The original version of this article unfortunately contained a mistake. The presentation of Fig. 1 and 2 was incorrect. The corrected Figs. 1 and 2 are given below. The authors apologize for the mistake. 


$$
\underline{\underline{1}}
$$



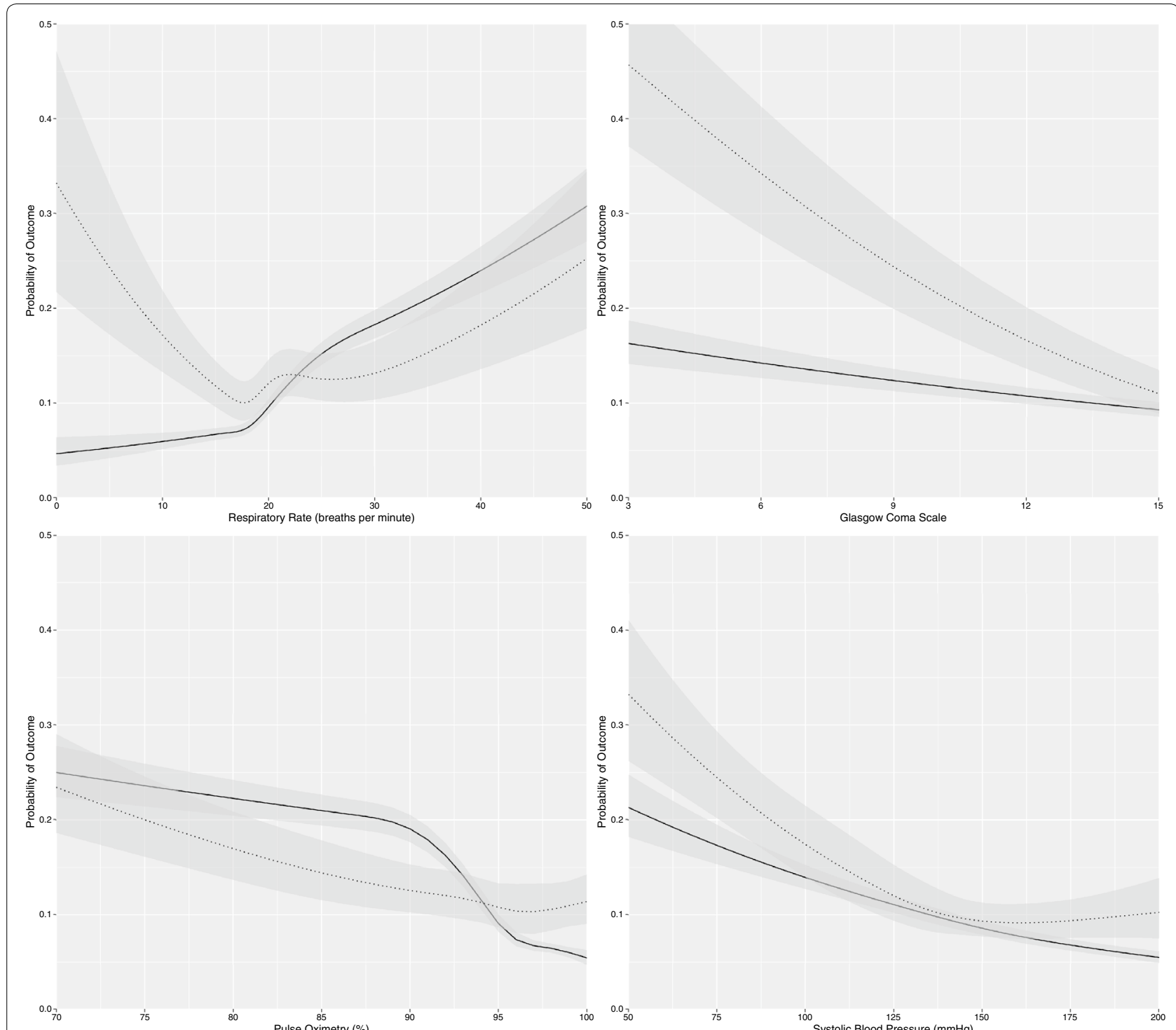

Fig. 2 Probability of infection diagnosis (solid line), or sepsis among patients with infection (dotted line), with 95\% confidence bands for select patient measures on the original measurement scale. Note sepsis diagnosis includes patients not discharged from the ED that had any sepsisrelated organ dysfunction ICD10-CA diagnosis or procedure code in the ED record

\section{Author details}

${ }^{1}$ Institute of Health Policy, Management and Evaluation, Dalla Lana School of Public Health, University of Toronto, Health Sciences Building, 155 College Street, Suite 425, Toronto, ON M5T 3M6, Canada. ${ }^{2}$ Rescu, Li Ka Shing Knowledge Institute, St. Michael's Hospital, Toronto, ON, Canada. ${ }^{3}$ Department of Critical Care Medicine, Sunnybrook Health Sciences Centre, Toronto, ON, Canada. ${ }^{4}$ Interdepartmental Division of Critical Care, University of Toronto, Toronto, ON, Canada. ${ }^{5}$ Department of Anesthesia, University of Toronto, Toronto, ON, Canada. ${ }^{6}$ Division of Emergency Medicine, Department of Family and Community Medicine, University of Toronto, Toronto, ON, Canada. ${ }^{7}$ Sunnybrook Centre for Prehospital Medicine, Sunnybrook Health Sciences Centre, Toronto, ON, Canada. ${ }^{8}$ Division of Emergency Medicine, Department of Medicine, University of Toronto, Toronto, ON, Canada. ${ }^{9}$ Cumming School of Medicine, University of Calgary, Calgary, AB, Canada.

\section{Publisher's Note}

Springer Nature remains neutral with regard to jurisdictional claims in published maps and institutional affiliations.

Published online: 30 June 2020 